\title{
La problemática de los canes vagabundos y los planes de control a nivel de gobiernos locales en Lima - Perú
}

\author{
The problem of stray dogs and control plans at the local government level \\ in Lima, Peru
}

\author{
Sofía Cáceda ${ }^{1}$, Daphne León ${ }^{1}$, Néstor Falcón ${ }^{1}$
}

\section{Resumen}

\begin{abstract}
El objetivo del estudio fue determinar la importancia de la presencia de canes vagabundos dada por los gobiernos locales de Lima Metropolitana e identificar los planes de control propuestos. El estudio descriptivo involucró a 32 de las 43 municipalidades de Lima Metropolitana que aceptaron participar. Se utilizó el modelo de encuesta realizado por la OIE respecto al control de la población de canes y canes vagabundos. El 90.6\% (29) consideraba a los canes vagabundos como un problema en su distrito, siendo la reproducción no controlada el problema más grave. El 58.6\% (17) indicó que la comuna no contaba con un plan que aborde la problemática, mientras que 31\% (9) contaba con uno y $10.4 \%$ (3) no lo tenía, pero realizaba actividades para enfrentarlo. Los métodos quirúrgicos (esterilizaciones y castraciones) fueron los más mencionados por las autoridades para tratar de controlar la población de canes vagabundos, mientras que la eutanasia solo fue mencionada para casos de enfermedad terminal o agresividad. El 59.4\% (19) mencionó que la municipalidad no contaba con presupuesto destinado al área de salud pública veterinaria; asimismo, el presupuesto insuficiente o inexistente $(82.1 \%)$ y la falta de infraestructura para el desarrollo de actividades (71.4\%) fueron las principales limitantes del municipio para abordar la problemática de los canes vagabundos. La información obtenida puede servir para tomar consciencia sobre el impacto de los canes vagabundos en la salud pública y valorar la necesidad de elaborar planes de control que se ajusten a las necesidades y limitaciones de cada distrito.
\end{abstract}

Palabras clave: mordeduras, perros, salud pública, zoonosis

\footnotetext{
${ }^{1}$ Laboratorio de Epidemiología y Salud Pública en Veterinaria, Facultad de Medicina Veterinaria y Zootecnia, Universidad Peruana Cayetano Heredia, Lima, Perú

${ }^{2}$ E-mail: sofia.caceda.m@upch.pe
}

Recibido: 9 de agosto de 2020

Aceptado para publicación: 2 de abril de 2021

Publicado: 23 de junio de 2021

CLos autores. Este artículo es publicado por la Rev Inv Vet Perú de la Facultad de Medicina Veterinaria, Universidad Nacional Mayor de San Marcos. Este es un artículo de acceso abierto, distribuido bajo los términos de la licencia Creative Commons Atribución 4.0 Internacional (CC BY 4.0) [https:// creativecommons.org/licenses/by/4.0/deed.es] que permite el uso, distribución y reproducción en cualquier medio, siempre que la obra original sea debidamente citada de su fuente original 
The aim of this study was to determine the importance of stray dogs by the local governments of Metropolitan Lima and to identify the proposed control plans. The descriptive study involved 32 of the 43 municipalities of Metropolitan Lima that agreed to participate. The survey model carried out by the OIE regarding the control of dog population was used. In general, 90.6\% (29) considered stray dogs a problem in their district, being uncontrolled reproduction the most serious issue. In addition, $58.6 \%$ (17) indicated that the municipality did not have a plan to address the problem, while $31 \%$ (9) had a plan and $10.4 \%$ (3) did not but carried out activities to address it. Surgical methods (spaying and neutering) were the most mentioned by the authorities to try to control the stray dog population, while euthanasia was only mentioned in cases of terminal illness or aggressiveness. Regarding budget allocation, 59.4\% (19) indicated that the municipality did not have specific budget for the veterinary public health area; likewise, the insufficient or non-existent budget $(82.1 \%)$ and the lack of infrastructure for the development of activities $(71.4 \%)$ were the main limitations of the municipalities to address the problem of stray dogs. The information obtained can be used to raise awareness about the impact of stray dogs on public health and assess the need to develop control plans that are adjusted to the needs and limitations of each district.

Key words: bites, stray dogs, public health, zoonosis

\section{INTRODUCCIÓN}

Los canes vagabundos son aquellos que no se encuentran bajo control directo de una persona o entidad y por tanto deambulan libremente. La OIE (2019) clasifica a este tipo de canes bajo las siguientes categorías: vagabundos con dueño, vagabundos sin dueño y asilvestrados. Los canes vagabundos son atribuidos como responsables de la transmisión de enfermedades zoonóticas, que afectan principalmente a personas inmunocomprometidas (Steele, 2008; Stull et al., 2015). Además, generan contaminación ambiental debido a los residuos de basura y excreciones que dejan en parques y vías públicas (León et al., 2013) y que pueden ser fuente de infección de parasitosis para las personas, como es el caso de la toxocariasis (Chávez et al., 2002).

Los canes vagabundos son responsabilizados también, de ocasionar accidentes por mordedura que producen lesiones físicas, secuelas emocionales y estéticas, transmisión de enfermedades y complicaciones infecciosas secundarias (Glausiuss et al., 2000). Asimismo, a estos animales se les responsabiliza de ocasionar accidentes de tránsito que ponen en riesgo la vida de las personas y afectan su propio bienestar por falta de cuidados veterinarios requeridos posterior al accidente (Álvarez y Domínguez, 2001; Canal et al., 2018; Voslárová y Passantino, 2012).

Dada la importancia otorgada a los canes vagabundos sobre la salud pública en Perú, en el año 2001 se aprobó la Ley N. ${ }^{\circ}$ 27596 que regula el «Régimen Jurídico de Canes» que tiene la finalidad de salvaguardar la integridad, salud y tranquilidad de las personas y en el año 2002 se aprobó su reglamento (D.S. N. $\left.{ }^{\circ} 006-2002\right)$. Posteriormente, en el año 2016 se aprobó la Ley N. ${ }^{\circ} 30407$, «Ley de Protección y Bienestar Animal» que tiene como finalidad garantizar el bienestar y la protección de todas las especies de animales vertebrados domésticos o silvestres man- 
tenidos en cautiverio, en el marco de las medidas de protección de la vida, la salud de los animales y la salud pública. Estas normas son de aplicación nacional y de cumplimiento obligatorio para las autoridades sectoriales, regionales y locales. Estos últimos, al ser organismos con autonomía política, económica y administrativa, tienen la competencia para aplicar las disposiciones de la ley a través de ordenanzas municipales.

Estas leyes señalan los deberes y derechos de los propietarios, así como las sanciones respectivas que emanan de las mismas, en caso de su incumplimiento (Robles et al., 2017). Ambas normas abordan el tema de abandono de los animales, evento que se encuentra relacionado a la presencia de canes vagabundos. En este contexto, el objetivo del estudio fue determinar la importancia que otorgan los gobiernos locales de Lima Metropolitana a la presencia de canes vagabundos en sus distritos e identificar los planes de control propuestos y en ejecución.

\section{Materiales y Métodos}

El estudio de tipo observacional descriptivo se realizó en la ciudad de Lima Metropolitana. La población objetivo fueron las autoridades encargadas del programa relacionado a Tenencia Responsable de Animales de Compañía (TRAC) de las 43 municipalidades distritales que forman parte de Lima Metropolitana.

Se utilizó como instrumento de recolección de información el formulario utilizado por la OIE respecto al control de la población de canes y canes vagabundos (Dalla et al., 2010), el cual fue modificado parcialmente en forma de acuerdo a las sugerencias ofrecidas por profesionales especialistas en el tema, con la finalidad de facilitar su entendimiento y aplicación. El instrumento contó una sección respecto a información general sobre el control de la población canina y otra específica sobre canes vagabundos, las cuales fueron:
I. Información general sobre el control de la población canina. Incluía si la municipalidad contaba con:

- Programa de control de la población canina.

- Ordenanza que obligue a los vecinos a registrar a sus canes de forma obligatoria.

- Ordenanza que obligue a la identificación canina, el tipo de identificación requerida y si es que consideraba multa por el incumplimiento del registro $\mathrm{y} / \mathrm{o}$ identificación canina.

- Presupuesto asignado al área de veterinaria o salud pública veterinaria.

II. Información sobre los canes vagabundos

- $\quad$ Si consideraban que los canes vagabundos representaban un problema.

- La valoración acerca de los principales problemas relacionados a la presencia de canes vagabundos (accidentes por mordedura, transmisión de enfermedades zoonóticas, contaminación ambiental, contaminación sonora, reproducción no controlada, y otros que pudiera mencionar el entrevistado).

- La municipalidad distrital contaba con algún plan oficial que aborde la problemática de los canes vagabundos y medidas de control ante esto (métodos de control utilizado y una evaluación específica para definir el uso de la eutanasia).

- Disponibilidad de albergue municipal para canes vagabundos que incluía cantidad de perros albergados y reinsertados a la sociedad mediante programa de adopción.

- Limitaciones del municipio para abordar la problemática de los perros vagabundos.

Se visitó a las autoridades municipales encargadas de los programas relacionados a TRAC de los distritos de Lima Metropolitana con el fin de explicarles el propósito del estudio y solicitarles su participación. Para esto, los representantes de las municipalidades firmaron el consentimiento de participa- 
ción y se les entregó el instrumento de recolección de la información y las instrucciones para su llenado. El instrumento, modificado en forma, constó de preguntas abiertas de respuestas puntuales (información cuantificable y especificación en caso de respuestas con opciones «otros») y preguntas cerradas con opciones binarias (sí / no), de valoración mediante escala de Likert y de alternativas múltiples en las cuales los participantes podrían marcar más de una opción cuando correspondía.

Los participantes tuvieron a disposición el instrumento una semana a fin de que el llenado del documento se realizara con el cuidado correspondiente, especialmente en los casos que se requiriera realizar búsqueda de información complementaria, a fin de obtener información fidedigna. Después de dicho periodo, el investigador recogió el instrumento como respuesta al llamado de la autoridad participante.

La información obtenida en estos formularios fue transferida a una hoja de cálculo de Microsoft Excel y fue resumida mediante estadística descriptiva utilizando valores absolutos y relativos para expresar los resultados de cada una de las categorías de las variables incluidas en el estudio. Los resultados acerca de presupuestos, importancia que se le da a los perros vagabundos y disponer de albergues temporales fueron clasificados de acuerdo con los niveles socioeconómicos (NSE) predominantes en los distritos $(\mathrm{A}=$ alto; $\mathrm{B}=$ medio alto; $\mathrm{C}=$ medio; $\mathrm{D}=$ medio bajo; $\mathrm{E}=$ bajo), según información del Instituto Nacional de Estadística e Informática (INEI, 2017).

El estudio fue aprobado por el Comité Institucional de Ética de la Universidad Peruana Cayetano Heredia mediante Constancia 371-17-19. La participación en el estudio fue voluntaria y se mantuvo en reserva la identidad de las personas e instituciones a las que representaban.

\section{Resultados}

Se recolectó información del 74.4\% (32/ 43) de las municipalidades de Lima Metropolitana. Las personas que respondieron las encuestas fueron principalmente los médicos veterinarios $(46.9 \% 15 / 32)$ como autoridades encargadas de desarrollar las actividades relacionadas a TRAC en las municipalidades participantes; sin embargo, profesionales de otras disciplinas también fueron los responsables de responder la encuesta, entre ellos Enfermería ( $\mathrm{n}=3)$, Administración (2), Técnico de Administración (2), y Contabilidad, Ingeniería Forestal, Medicina Humana, Odontología, Psicología, Secretariado, y carreras técnicas de computación, producción pecuaria y de sanidad (uno por disciplina)

El 71.9\% (23/32) de las municipalidades participantes indicaron contar con algún programa de control de la población de canes y el 75\% (24/32) indicó tener una norma que obliga a los propietarios de los canes a registrarlos en la municipalidad. De estos últimos, el 75\% (18/24) mencionó que la identificación de los canes resultaba obligatoria por mandato de la ordenanza municipal del distrito. Entre las 18 municipalidades que respondieron acerca del tipo de identificación aceptada, se encontró que predominaban aquellos que correspondían a un tipo de documento manual, como el denominado documento nacional de identidad canino («DNI canino»), carné o ficha de registro municipal (Cuadro 1).

De las 24 municipalidades que indicaron que el registro o identificación de canes era obligatorio por normativa municipal, el $91.7 \%$ (22/24) mencionó la existencia de multas por el incumplimiento de dicha obligación. Asimismo, el 59.4\% (19/32) del total de municipalidades mencionó que la institución no contaba con un presupuesto asignado al área de veterinaria o salud pública veterinaria, en tanto que $5 / 9(55.7 \%)$ de los distritos donde predominaban los NSE A/B y 8/32 (34.8\%) de los que predominaban los NSE $\mathrm{C} / \mathrm{D}$ contaban con dicho presupuesto. 
Cuadro 1. Tipo de identificación aceptada dentro de la ordenanza municipal en 18 de 43 distritos de Lima Metropolitana (2019)

\begin{tabular}{lcc}
\hline Identificación & $\mathrm{n}$ & $\%$ \\
\hline $\begin{array}{l}\text { DNI canino / carné / } \\
\text { registro municipal }\end{array}$ & 13 & 72.2 \\
Microchip & 7 & 38.9 \\
Placa & 5 & 27.8 \\
\hline
\end{tabular}

La municipalidad podía ofrecer más de una respuesta

E1 90.6\% (29/32) de las municipalidades mencionaron que los canes vagabundos representaban un problema en su distrito. Los tres distritos ajenos a este problema correspondieron a distritos con predominio de NSE
A/B. La distribución de la respuesta acerca de la percepción de la importancia de los problemas que pueden ocasionar los canes vagabundos se presenta en la Figura 1.

Entre los distritos que indicaron que los canes vagabundos eran un problema, el $58.6 \%(17 / 29)$ mencionó que la municipalidad no contaba con un plan oficial para abordar la problemática de los canes vagabundos, el 10.4\% (3/29) indicó que no contaban con uno, pero realizaban actividades que buscaban enfrentar ese problema, mientras que el 31\% (9/29) mencionó que tenía dicho plan de trabajo.

De 10 municipalidades que mencionaron métodos de control utilizados en la población de canes vagabundos, todos mencionaron intervenciones quirúrgicas a los animales capturados (esterilización y castración), seis de ellos se refirieron además a las adopciones, dos a la captura/internamiento y dos aplicarían eutanasia, condicionalmente, en caso de enfermedad terminal y presencia de canes agresivos.

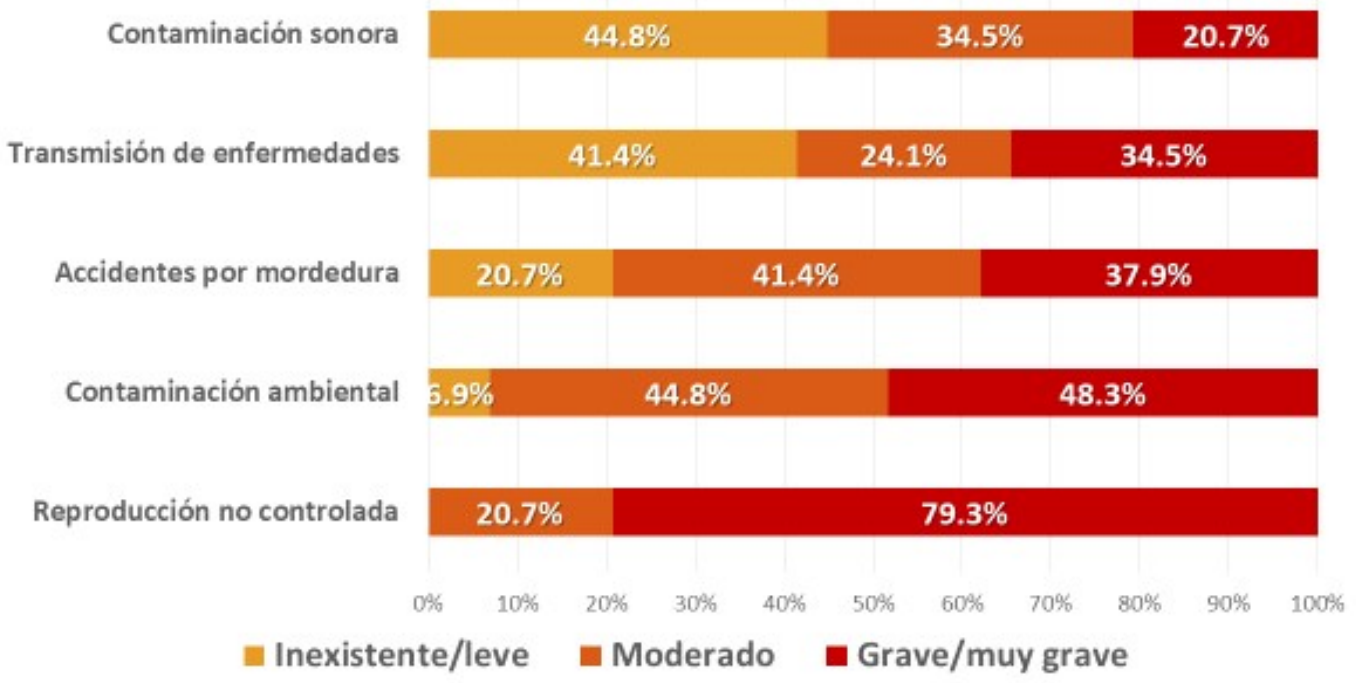

Figura 1. Percepción de las municipalidades participantes en el estudio acerca de la importancia de los problemas que pueden ocasionar los canes vagabundos. Lima, $2019(\mathrm{n}=29)$. 
Cuadro 2. Percepción de las autoridades municipales acerca de las limitantes del municipio para abordar la problemática de los canes vagabundos en 28 de 43 municipalidades de Lima Metropolitana, 2019

\begin{tabular}{lcc}
\hline \multicolumn{1}{c}{ Limitantes } & $\mathrm{n}$ & $\%$ \\
\hline Presupuesto insuficiente o inexistente & 23 & 82.1 \\
Falta de infraestructura para el desarrollo de actividades & 20 & 71.4 \\
Falta de planes, programas, proyectos o propuestas & 11 & 39.3 \\
Problemas con grupos animalistas & 9 & 32.1 \\
Falta de personal capacitado & 9 & 32.1 \\
Ausencia de información del abandono de canes en calles & 8 & 28.6 \\
Desinterés de diferentes actores & 6 & 21.4 \\
\hline
\end{tabular}

Se podía ofrecer más de una respuesta por encuesta

Solo $9.4 \%(3 / 32)$ de las autoridades encuestadas mencionaron tener albergues temporales municipales y todos ellos correspondieron a distritos donde predominaba el NSE A/B. De estas, dos indicaron que albergaron 50 y 85 canes entre enero y noviembre de 2019 , y que el 40 y $82.4 \%$ fueron adoptados, respectivamente.

El presupuesto insuficiente o inexistente para el desarrollo de actividades que permitan abordar la problemática de los canes vagabundos fue la limitante mencionada con mayor frecuencia por los entrevistados. El detalle acerca de esta y otras razones se presenta en el Cuadro 2.

\section{Discusión}

El estudio buscó obtener una visión general sobre la situación actual de las políticas municipales de los distritos de Lima Metropolitana en cuanto a la problemática de los canes vagabundos y el control de la población canina en general. Esta problemática empezó a visualizarse, de forma focalizada, en estudios como los de Ochoa et al. (2014) y Granda (2018) al investigar la presencia de canes vagabundos en los distritos de Los Olivos y San Borja, respectivamente, a los que se sumó el estudio de Chávez et al. (2016), quienes estudiaron la presencia de canes vagabundos en mercados de Villa el Salvador.

La Ley que Regula el Régimen Jurídico de Canes (Ley N. ${ }^{\circ}$ 27596) encarga a las municipalidades la responsabilidad de los canes vagabundos, siguiendo el mandato de la OIE (2019) que en su capítulo 7.7 del Código Sanitario para los Animales Terrestres indica que los gobiernos locales asumen la responsabilidad de desarrollar y hacer cumplir la legislación relacionada a la tenencia de canes y el control de canes vagabundos. Para el caso de Perú, las municipalidades hacen efectivos los mandatos de la Ley N. ${ }^{\circ} 27596$ a través de las ordenanzas municipales, que en la actualidad existen en la mayoría de las municipalidades de Lima Metropolitana, a excepción de tres distritos (Santa Rosa, Punta Negra y Villa el Salvador). 
Alrededor de la mitad de las autoridades municipales responsables de los programas municipales relacionados a TRAC que participaron en el estudio resultaron ser médicos veterinarios. Su participación en la elaboración de planes de control de la población canina resulta fundamental, debido a que por su formación y competencia profesional en salud animal y salud pública se encuentran capacitados para desarrollar actividades para afrontar la problemática relacionada a los temas de TRAC, bajo el concepto de «Una Salud» (Terrada, 2018).

Las municipalidades participantes, que contaban con un programa de control de la población canina, lo hacían a través de la veterinaria municipal del distrito o a través de campañas periódicas en diferentes zonas de la comunidad. En la realización de estas campañas, algunas municipalidades contaban con la participación de ONGs y entidades privadas que brindaban su apoyo. La OIE (2019) menciona que el trabajo conjunto de las municipalidades y las ONGs es importante en los temas de educación sobre TRAC y que esta colaboración resulta común en los programas de control de la población canina. No obstante, la mayoría de los programas municipales no incluyen a los canes vagabundos, ya que las actividades que realizan están dirigidas a canes con dueño.

La implementación de las actividades de control de los canes vagabundos requiere de personal capacitado e infraestructura adecuada para la captura, transporte y contención de estos animales, limitantes que son mencionadas por las autoridades municipales en este estudio. Robles et al. (2017) habían identificado estas limitaciones años atrás, pero todo indica que pese al tiempo transcurrido no se han podido superar.

Las municipalidades de Lima Metropolitana que contaban con alguna ordenanza con relación a la Ley N. ${ }^{\circ} 27596$ mencionan que el registro e identificación de canes no se aplica tal como lo establecen estas ordenanzas. Para la mayoría, el tipo de identificación requerida es el «DNI canino» o «carné municipal». La identificación de los canes resulta importante para diferenciar a los animales vagabundos con y sin dueño. En ese sentido, los microchips ofrecen mayor eficiencia en caso de que los canes que se encuentren deambulando tengan dueño, como sería el caso de canes extraviados (García, 2009), donde los microchips permitirían ubicar al dueño y facilitar el retorno del animal a su hogar. Asimismo, permitiría identificar a los propietarios que permiten deambular libremente a sus animales y facilitaría la aplicación de las sanciones que mencionan las ordenanzas.

Más de la mitad de las municipalidades en las que predominaban los NSE A/B mencionaron contar con un presupuesto asignado al área de veterinaria o salud pública veterinaria. El NSE está asociado a la disposición de recursos económicos que podrían ser asignados para este tema, lo que se refleja en el nivel de avance de los programas, proyectos o actividades relacionadas a la TRAC aplicados en los distritos. Solo tres distritos, pertenecientes al NSEA/B, indicaron que los canes vagabundos no representaban un problema en su jurisdicción, sin que ello indique que no existan. Así, Granda (2017) encuentra que, en el distrito de San Borja, donde predomina el NSE A/B, se encontró un promedio de 2.1 y 2.6 canes $/ \mathrm{km}^{2}$ que deambulaban libremente en el día y la noche, respectivamente. En contraste, en el distrito de Los Olivos, donde predomina el NSE C/ $\mathrm{D}$, Ochoa et al. (2014) reportaron un promedio de 19.2 y 12.5 canes $/ \mathrm{km}^{2}$ para el día y la noche, respectivamente, lo que demostraría la relación inversa entre el NSE y el número de canes vagabundos.

Según la opinión de los participantes, la reproducción no controlada de los canes vagabundos representa el problema más importante. Esto podría ser un indicativo de que la capacidad reproductiva de estos canes no se ve afectada, debido a la disponibilidad de alimento que encuentran en la basura acumulada alrededor de los mercados y en las bolsas 
de basura dejadas en las vías públicas para que sean recogidas por el servicio de saneamiento ambiental (Álvarez y Domínguez, 2001). Las barreras físicas que impidan el acceso de canes a los mercados y hacia los depósitos donde se apila la basura en el exterior representa un factor fundamental para evitar el acceso a fuentes de alimento (Chávez et al., 2016).

La contaminación ambiental fue considerada el segundo problema de importancia. Cinquepalmi et al. (2012) mencionaron que la contaminación ambiental por heces de canes vagabundos representa un factor de riesgo en la transmisión de enfermedades. El tercer problema en importancia, mencionado por las municipalidades, fue los accidentes por mordedura. En este sentido, Morales et al. (2011) encontraron que un tercio de los accidentes por mordedura se produjo en la vía pública y estarían asociados a la presencia de canes vagabundos. Por su parte, Glausiuss et al. (2000) mencionan que estos accidentes, además de ocasionar lesiones físicas y secuelas psicológicas, contribuyen en la transmisión de enfermedades zoonóticas, siendo la rabia una de las principales. Por último, un menor problema se debe a la contaminación sonora, la cual provoca un impacto sobre la salud pública (Oguntunde et al., 2019).

La reproducción descontrolada de canes vagabundos representa un problema que debe ser afrontado por las autoridades municipales. Si bien existen diversos métodos, la esterilización y castración son los métodos más valorados, debido a su practicidad, seguridad, rapidez y menor efecto colateral (Pelaez et al., 2018). Además, estos métodos anulan de manera definitiva la capacidad reproductiva de los animales. Asimismo, las hembras esterilizadas a una temprana edad presentan menor riesgo de desarrollar piometras, tumores mamarios o alguna otra neoplasia relacionada, mientras que en los machos castrados disminuyen las posibilidades de neoplasias prostáticas y, además, exis- ten beneficios sobre el comportamiento (McKenzie, 2010).

Las esterilizaciones en hembras requieren de mayor asepsia y presentan un costo más elevado que las castraciones en machos, lo que puede representar una limitante en los programas de control de la población canina (Pelaez et al., 2019). No obstante, dado que unas de las principales limitantes indicadas por las autoridades municipales era la falta de presupuesto destinado al control de canes vagabundos y la falta de infraestructura adecuada, un programa de control basado en esterilizaciones se vería afectado si no se considera un aumento de los recursos económicos y la implementación de infraestructura que asegure cobertura suficiente para detener el crecimiento poblacional.

La mayor parte de las municipalidades participantes indicaron que no cuentan con un plan oficial para abordar la problemática de los canes vagabundos. La mayoría de las ordenanzas municipales indica que los canes vagabundos deben de ser recogidos por personal municipal para ser puestos a disposición del sector salud o en albergues, donde su tiempo de mantenimiento es de 30 días, tiempo en el cual se ha de procurar la adopción o custodia de los canes y de no lograrlo deberán de ser sacrificados. No obstante, Robles et al. (2017) señalan que estas actividades no suelen realizarse ante la falta de personal e infraestructura para la retención de canes vagabundos y a la falta de presupuesto y logística destinada para ello.

La ausencia de un albergue municipal es una razón por la que no se desarrollan las actividades de recojo de canes vagabundos. Dalla et al. (2010) mencionan que la implementación y mantenimiento de estos implican un costo que muchos países no pueden permitirse, siendo este el caso de la mayoría de los distritos de Lima Metropolitana. Por su parte, FAO (2014) menciona que los albergues representan una medida transito- 
ria para los canes vagabundos; sin embargo, no pueden considerarse una solución a largo plazo, debido a que no afrontan las causas que conllevan al abandono. Por esta razón, Fielding y Mather (2000) señalan que es esencial el aspecto educativo en cuanto a la TRAC, ya que, a través de la educación, el comportamiento humano puede ser modificado.

La Ley N. ${ }^{\circ} 27596$ menciona la necesidad de implementar campañas educativas para sensibilizar a la población respecto a los temas de TRAC y los contenidos de la ley, donde se señala las responsabilidades de los propietarios de los canes y las sanciones a las que se exponen las personas al no cumplir con la ley. Estas actividades pueden ser coordinadas con otras instituciones (Ministerio de Salud, Ministerio de Educación, etc.) para tener un mayor alcance e impacto. La OIE (2019) establece que la educación sobre tenencia responsable debe abordar la importancia de la selección de los canes y su cuidado para garantizar su bienestar; asimismo, reforzar la importancia del registro y uso de identificación canina, la prevención de enfermedades zoonóticas, el impacto negativo sobre la sociedad y el control reproductivo de estos canes.

Las municipalidades participantes indicaron la falta de presupuesto como la mayor limitante para abordar la problemática de los canes vagabundos. En ese sentido, la FAO (2014) señala que esto se puede atribuir a que, a comparación de otros problemas sociales, el problema de los canes vagabundos no representa una prioridad y que las autoridades suelen reaccionar solo en casos donde la economía se encuentre afectada.

Valencia (2012) realizó un estudio sobre las técnicas de control de la población canina alrededor del mundo, encontrando que el impacto de la legislación, a pesar de ser crucial en la lucha contra la problemática de los canes vagabundos, varía de acuerdo con la educación y cultura de los países. Así, las ONGs protectoras de animales en el Reino
Unido realizan campañas de tenencia responsable que han generado un impacto positivo en la sociedad y ha obligado al gobierno a preocuparse por este tema. Suecia presenta legislación básica; sin embargo, la población considera a los canes como parte de su familia y se preocupa por su bienestar; en tanto que Costa Rica, Panamá y Brasil cuentan con una ley, pero su aplicación resulta casi inexistente (Trent et al., 2005).

El abordaje del estudio respecto a la problemática de los canes vagabundos en Lima Metropolitana desde la perspectiva de las autoridades municipales encargadas resulta importante, ya que se recibe información directa de los responsables de enfrentar el problema. Sin embargo, a ello, se deben sumar otros temas que permitan visualizar la problemática en su real dimensión y permita buscar alternativas para enfrentarlas. La estimación de la población de canes, el conocimiento de las características de la crianza de los canes y otros aspectos sociales relacionados con los mismos para cada distrito de Lima Metropolitana representan herramientas de utilidad para conocer la magnitud del problema. De este modo, se podrá elaborar un programa de control de la población de canes que se ajuste a cada distrito y a las necesidades y limitaciones de cada localidad.

\section{Conclusiones}

- Menos del 50\% de las municipalidades participantes contaban con un médico veterinario a cargo de los temas relacionados a tenencia responsable de animales de compañía y ninguna contaba con un área exclusiva para el desarrollo de actividades relacionadas a la salud pública veterinaria.

- Las autoridades municipales reconocieron, en la mayoría de los distritos, que los canes vagabundos representan un problema y que su reproducción no controlada era la preocupación de mayor gravedad. 
- La falta de presupuesto e infraestructura adecuada fueron las principales razones mencionadas como limitantes para el control de los canes vagabundos.

- Solo un tercio de las municipalidades contaba con un plan oficial que abordaba la problemática de los canes vagabundos y quienes desarrollaban campañas de control reproductivo de dicha población utilizaban métodos quirúrgicos para la castración de los canes.

- Los espacios para mantener en custodia a los canes vagabundos (albergues municipales) solo se encontraron en tres distritos pertenecientes a los niveles socioeconómicos altos.

\section{Literatura Citada}

1. Álvarez E, Domínguez J. 2001. Programa para el control de integral de la población canina. AMMVEPE 12: 83-91.

2. Canal D, Martín B, de Lucas, Ferrer M. 2018. Dogs are the main species involved in animal-vehicle collisions in southern Spain: daily, seasonal and spatial analyses of collisions. Plos One 13: e0203693. doi: 10.1371/journal.pone.0203693

3. Chávez A, Casas E, Serrano M, Cajas J, Velarde J, La Rosa V, López T. 2002. Riesgo de contraer enfermedades parasitarias en los parques públicos de Lima y Callao. Rev Inv Vet Perú 13: 84-91. doi: 10.15381/rivep.v13i2.7337

4. Chávez C, Falcón N, León D, Sánchez D. 2016. Canes vagabundos en el interior y alrededores de mercados formales de Villa el Salvador, Lima, Perú. Rev Inv Vet Perú 27: 176. doi: 10.15381/ rivep.v27i1.11456

5. Cinquepalmi V, Monno R, Fumarola $L$, Ventrella G, Calia C, Greco MF, Vito Dd, Soleo L. 2012. Environmental contamination by dog's faeces: a public health problem? Int J Environ Res $\mathrm{Pu}$ 10: 72-84. doi: 10.3390/ijerph10010072
6. Congreso de la República de Perú. 2001. Ley $\mathrm{N}^{\circ} 27596$ - Ley que regula el Régimen Jurídico de Canes. Diario Oficial El Peruano. Lima, Perú, 14 de diciembre de 2001.

7. Congreso de la República de Perú. 2016. Ley $\mathrm{N}^{\circ} 30407$ - Ley de Protección y Bienestar Animal. Diario Oficial El Peruano. Lima, Perú, 8 de enero de 2016.

8. Dalla P, Kahn S, Stuardo L, Iannetti L, Di Nardo A, Serpell J. 2010. Freeroaming dog control among OIEmember countries. Prev Vet Med 97: 5863. doi: 10.1016/j.prevetmed.2010.07.001

9. [FAO] Food and Agriculture Organization. 2014. Dog population management. Report of the FAO/ WSPA/IZSAM expert meeting. Rome: FAO. [Internet]. Available in: http:// www.fao.org/3/a-i4081e.pdf

10. Fielding W, Mather J. 2000. Stray dogs in an island community: a case study from New Providence, The Bahamas. Prev Vet Med 3: 305-319. doi: 10.1207/ S15327604JAWS0304_3

11. García A. 2009. Bases para el establecimiento de un método de identificación de perros en la comuna de Maipú. Tesis de Médico Veterinario. Santiago de Chile: Univ. de Chile. 90 p.

12. Glausiuss G, Ascione I, Sehabiague G 2000. Mordeduras por animales en la edad pediátrica. Arch Pediatr Urug 71: 24-30.

13. Granda D. 2018. Estimación de la población de canes vagabundos en el distrito de San Borja, 2017. Tesis de Médico Veterinario Zootecnista. Lima-Perú: Univ, Peruana Cayetano Heredia. 23 p.

14. [INEI] Instituto Nacional de Estadística e Informática. 2017. Planos estratificados de Lima Metropolitana a nivel de manzanas 2020, según ingreso per cápita del hogar. 74.p [Internet]. Disponible en: https://www.inei.gob.pe/media/MenuRecursivo/publicaciones_digitales/Est/Lib1744/libro.pdf 
15. Leoìn D, Panta S, Yarlequeì C, Falcoìn N. 2013. La convivencia con mascotas en zonas periurbanas: Experiencia en Lima - Peruì. MV Rev Cien Vet 29: 21-25.

16. McKenzie B. 2010. Evaluating the benefits and risks of neutering dogs and cats. CAB Reviews Perspectives in Agriculture, Veterinary Science, Nutrition and Natural Resources 5. doi: 10.1079/pavsnnr20105045

17. Morales C, Falcón N, Hernández H, Fernández C. 2011. Accidentes por mordedura canina, casos registrados en un hospital de niños de Lima, Perú 1995 - 2009. Rev Peru Med Exp Salud Publica 28: 639-642. doi: 10.1590/s172646342011000400011

18. Ochoa Y, Falcón N, Zuazo J, Guevara B. 2014. Estimación de la población de perros callejeros en el distrito de Los Olivos, Lima, Perú. Rev Inv Vet Perú 25: 366-373. doi: 10.15381/rivep.v25i3.10114

19. Oguntunde P, Okagbue H, Oguntunde O, Odetunmibi O. 2019. A study of noise pollution measurements and possible effects on public health in Ota Metropolis, Nigeria Open Access Maced J Med Sci 7: 1391-1395. doi: 10.3889/ oamjms.2019.234

20. [OIE] World Organization for Animal Health. 2019. Terrestrial Animal Health Code. $28^{\text {th }}$ ed. Paris: OIE. [Internet]. Available in: https://www.oie.int/standardsetting/terrestrial-code/access-online/

21. Pelaez M, Echevarría L, Soler-Tovar D, Falcón N. 2019. Métodos de contracepción en el control poblacional de perros: un punto de vista de los médicos veterinarios de clínica de animales de compañía. Salud Tecnol Vet 6: 55-61. doi: 10.20453/stv.v6i2.3459

22. Robles R, León D, Falcón N. 2017. Evaluación del contenido y aplicabilidad de las ordenanzas municipales emitidas dentro del marco de la ley que regula el régimen jurídico de canes (Ley N ${ }^{\circ} 27596$ ) en los distritos de Lima Metropolitana. MV Rev Cien Vet 33: 5-12.

23. Steele R. 2008. Should immunocompromised patients have pets? Ochsner J 8: 134-139.

24. Stull J, Brophy J, Weese J. 2015. Reducing the risk of pet-associated zoonotic infections. Can Med Assoc J 187: 736-743. doi: 10.1503/cmaj. 141020

25. Terrada P. 2018. Evaluación de las actividades de prevención, vigilancia y control de las zoonosis prioritarias en Chile, bajo el enfoque» una salud». Tesis de Médico Veterinario. Santiago de Chile: Univ. de Chile. $138 \mathrm{p}$.

26. Trent N, Edwards S, Felt J, O'Meara $K$. 2005. International animal law, with a concentration on Latin America, Asia, and Africa. In: Salem DJ, Rowan AN (eds). The State of the Animals III: Washington DC, USA: Humane Society Press. p 65-77.

27. Valencia C. 2012. Técnicas de control de poblaciones caninas callejeras usadas a nivel mundial. Revisión bibliográfica. Tesis de Médico Veterinario. Valdivia, Chile: Univ. Austral de Chile. 26 p.

28. Voslárová E, Passantino A. 2012. Stray dog and cat laws and enforcement in Czech Republic and in Italy. Ann I Super Sanita 48: 97-104. doi: 10.4415/ ANN_12_01_16 